\title{
Preliminary Study of a Physiological Evaluation Method on Attentiveness Concentration during Mental Arithmetic; Correlation between Task Performance and Physiological Indices
}

\author{
Tatsuro Tanabe ${ }^{1)}$, Kosuke Ikemura ${ }^{2)}$, Hiroko Sawai ${ }^{3)}$ and Emi Koyama ${ }^{1)}$ \\ 1) Division of Design Engineering and Management, Graduate School of Science and Technology, \\ Kyoto Institute of Technology \\ 2) Division of Design Engineering and Management, Graduate School of Science and Technology, \\ Kyoto Institute of Technology, presently SEIWA Electric MFG. Co., Ltd. \\ 3) Division of Design Engineering and Management, Graduate School of Science and Technology, \\ Kyoto Institute of Technology, presently HINO Motors, Ltd.
}

\begin{abstract}
It is important that task performance is physiologically evaluated in consideration of arousal level. But there are relatively few preceding studies. In this study, the relationship between task performance and physiological indices was studied with regard to attentiveness concentration. The subjects were eight healthy college students. They performed calculations and a visual display terminal (VDT) task. Electroencephalogram (EEG) frequency component, alpha attenuation coefficient (AAC), skin potential level (SPL), blood flow of the finger tip skin (BF), and visual analog scale (VAS), were measured. In order to quantify task performance, correlations between the task performance and physiological indices during the mental task were analyzed. The results suggest that AAC correlates with the error rate in calculation. $\mathrm{BF}$ also correlates with the error rate in calculation, while the calculation speed correlates with SPL. It can be inferred that the task speed and error rate are supposed to be related to the different physiological background. J Physiol Anthropol 26(5): 553-557, $2007 \mathrm{http}: / /$ www.jstage.jst.go.jp/browse/jpa2
\end{abstract}

[DOI: 10.2114/jpa2.26.553]

Keywords: task performance, arousal level, attentiveness, physiological indices, blood flow, SPL, fatigue

\section{Introduction}

In recent years many brain-training products have been released. This is because cerebral-powered work operation efficiency is required in the social community and in daily life. In fact, good performance is required in many aspects of our life, for example, office work, and academic training. So far, however, comparatively little information has emerged on task performance as objects of physiological scientific research. Studies in humans through the use of PET, fMRI, EEG, MEG, etc. have quantified human brain functions. But they have revealed only the local existence of brain functions. Brain work performance and working efficiency are not adequately quantified. Researchers who had previously performed task performance studies were not aware of the potential existence of arousal level. It is difficult to quantify brain performance level; on the other hand, brain arousal level is not difficult to quantify. The Alpha Attenuation Test (AAT) is able to quantify arousal level (Michimori et al., 1994; Michimori et al., 1999), so it is important that the task performance is physiologically evaluated in consideration of arousal level (Moore-Ede, 1993). Arousal level is related to task performance (Yagi, 1997). There were the physiological indices that suggested correlation with task performance in the earlier studies. Kawasaki et al. (1994) indicate that skin potential level correlates with task performance. Also, Nishimura et al. (1980) reported that human skin potential level reflects vigilance level of the person. In addition, it is significant to measure plural physiological indices during the task. Fumoto (1977) measured blood flow of the finger skin and pulse rate during his experiments. He reported that attentiveness on a task is reflected better by blood flow of the finger skin than by pulse rate. Also Jin et al. (2005) stated that the beta-1 power ratio of an EEG is useful in the evaluation of mental workload as degree of difficulty, and HRV might be effective in evaluating the time change of a mental workload with accomplishment of the task. But there are few detailed studies. The purpose of this preliminary study is to investigate the relationships between the plural task performances and the plural physiological 
indices under conditions of attentiveness.

\section{Methods}

\section{Experimental condition}

The experiment was conducted over two months (from November to December 2005). The temperature of the measurement room was set at $24.6 \pm 1.3$ degrees Celsius. Horizontal illuminance on the desk was $512 \pm 121 x$. Vertical illuminance around the face while sitting on the chair was 126 $\pm 91 x$. Subjects were eight healthy college students (male: 7, female: 1, 23 \pm 1years). They were instructed to keep the schedule regularly from two days before to the final day of the experiment through the experimental period for approximately 4-6 days in order to control the arousal level of the experiment day nearly equally. Smoking, any drink containing caffeine, any drugs, and excessive physical exercise were restricted for two hours until the start of the experiment. Prior to participation in this study, the purpose and procedure of this study were explained in detail. Informed written consent was obtained from all subjects. Subjects received appropriate rewards., The experimental protocol was as shown in Fig. 1.

\section{Measurement items}

The electroencephalogram (EEG) was recorded at a frequency of $500 \mathrm{~Hz}$ and EEG frequency components were calculated on a personal computer through the use of a polymate system (TEAC Company). The Alpha Attenuation Coefficient (AAC) was evaluated from O1 or O2 of EEG. Skin Potential Level (SPL) during each task, was measured continuously at the palm, the top of the hand, and the wrist of the left hand (Techno Science Company). Blood flow of the finger tip skin (BF) during each task was measured continuously at the flat of a thumb using OMEGAFLO FLOC1 (Omega Wave, Inc). Subjective assessment was measured with a visual analog scale (VAS). The temperature of the room was measured using LT-8 (Gram Corp).

\section{Calculation task}

Subjects were instructed to make three-digit mental calculations sitting down on a chair in the measurement room for five minutes. Three-digit numbers were laid out on the test paper. The three-digit number shown on the paper was subtracted from 1000, and each digit number of the subtracted result was distinguished into "the largest," "the smallest," and "other". Then "the smallest" was subtracted from "the largest." This answer was multiplied by the "other". At the end of one question, only the final answer was written on the paper, not the calculation process. Mental calculations were divided up at one-minute intervals. Subjects were instructed to go to the next segment at one-minute intervals by a voice coming from a speaker. From this task, the calculation speed (number of answers/minute) and error rate (number of mistakes/number of answers) was calculated every one-minute period. Subjects were instructed not to move the body during the task as much

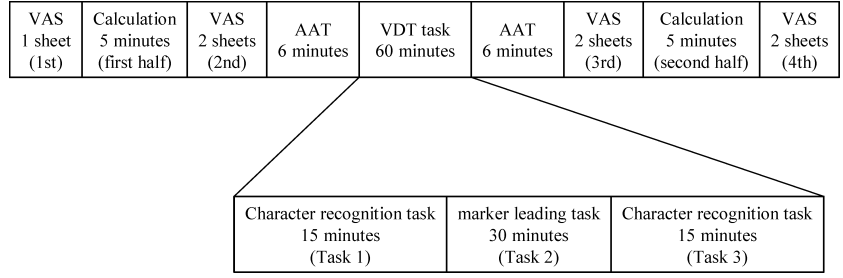

Fig. 1 Schedule of the experiment.

as possible. This task was attempted twice. The first attempt was made before the VDT task and second after the VDT task (Fig. 1).

\section{VDT task}

The VDT task was done for sixty minutes. In the first fifteen minutes (Task 1) and the last fifteen minutes (Task 3), subjects were instructed to find a certain character correctly among four kinds of characters on the display and count them. then click the mouse by the numbers just found. From this task, the count mistake rate was calculated (source number-count number) every one-minute period. For the middle thirty minutes (Task 2), subjects were instructed to rapidly lead a marker from start to goal not bumping against a wall in the maze on the display. From this task, the bump rate was calculated every one-minute period and the task completion speed was also calculated. Subjects were instructed not to move the body during the task as much as possible.

\section{Statistical analyses}

The mean values of each set of measurement data during each task are calculated as one trial average. Differences between groups (individual variability-number of days) were tested by a two-way ANOVA with * set at $p<0.05$ and ** set at $p<0.01$. The correlation between each item was determined by Spearman's correlation coefficient with * set at $p<0.05$ and $* *$ set at $p<0.01$. The correlation coefficient was calculated for all subject data.

\section{Results}

Differences between groups by a two-way ANOVA

Table 1 shows the significant individual differences and the significant day-by-day differences. There is a significant difference in the interindividual variability of all items except for BF in the second half. There is no significant difference in the number of days of the first half of calculation error, AAT and SPL.

Correlation between the number of days and task performance

Table 2 shows that number of days moderately correlates with the calculation speed (Spearman: first half: $r=0.595^{* *}$, second half: $r=0.525^{* *}$ ) but does not strongly correlate with error rate in mental calculation (first half: $r=-0.314$, second 
Table 1 Significant difference of the factor "individual variability" and "number of days"

\begin{tabular}{|c|c|c|c|c|c|c|c|}
\hline & \multicolumn{2}{|c|}{ Calculation speed } & \multicolumn{2}{|c|}{ Calculation error } & \multicolumn{3}{|c|}{ VDT task } \\
\hline & First & Second & First & Second & Task 1 & Task 2 & Task 3 \\
\hline Interndividual variability & $0.015 *$ & $0.0001 * *$ & $0.021 *$ & $0.008 * *$ & $0.0005 * *$ & $0.002 * *$ & $0.002 * *$ \\
\hline \multirow[t]{3}{*}{ Number of days } & $0.003 * *$ & $0.0001 * *$ & 0.451 & $0.036 *$ & $0.0001 * *$ & $0.040 *$ & $0.0001 * *$ \\
\hline & \multicolumn{2}{|c|}{ AAT } & \multicolumn{2}{|c|}{$\mathrm{BF}$} & \multicolumn{2}{|c|}{ SPL } & VAS(fatigue) \\
\hline & First & Second & First & Second & First & Second & $3 \mathrm{rd}$ \\
\hline Interindividual variability & $0.0001 * *$ & $0.0001 * *$ & $0.0007 * *$ & 0.242 & $0.014 *$ & $0.0001 * *$ & $0.0001 * *$ \\
\hline Number of days & 0.392 & 0.770 & $0.002 * *$ & $0.004 * *$ & 0.462 & 0.855 & $0.002 * *$ \\
\hline
\end{tabular}

$* * p<0.01 * p<0.05$

Note) Two-way ANOVA is used to compare means among groups.

Table 2 Correlation between the number of days and task performance

\begin{tabular}{|c|c|c|c|c|c|c|c|}
\hline & \multicolumn{2}{|c|}{ Calculation } & \multicolumn{3}{|c|}{ VDT task } & \multicolumn{2}{|c|}{ Calculation } \\
\hline & Speed first & Error first & Task 1 & Task 2 & Task 3 & Speed second & Erorr second \\
\hline $\begin{array}{l}\text { Correlation } \\
\text { coefficient }\end{array}$ & 0.595 & -0.314 & 0.660 & -0.045 & -0.479 & 0.525 & -0.380 \\
\hline$P$-value & $* *$ & & $* *$ & & $* *$ & $* *$ & $*$ \\
\hline
\end{tabular}

$* * p<0.01 * p<0.05$

Note) Spearman's Rank-Order Correlation Coefficient is calculated.

Table 3 Correlation between the VAS (fatigue) and task performance

\begin{tabular}{|c|c|c|c|c|c|c|c|}
\hline \multirow{2}{*}{ VAS (fatigue) } & \multicolumn{2}{|c|}{ Calculation } & \multicolumn{3}{|c|}{ VDT task } & \multicolumn{2}{|c|}{ Calculation } \\
\hline & Speed first & Error first & Task 1 & Task 2 & Task 3 & Speed second & Error second \\
\hline $1 \mathrm{st}$ & 0.053 & $-0.379 *$ & -0.030 & -0.191 & -0.019 & 0.113 & -0.143 \\
\hline $2 \mathrm{st}$ & 0.068 & -0.122 & 0.027 & 0.106 & 0.094 & 0.146 & -0.038 \\
\hline $3 \mathrm{st}$ & $0.605 * *$ & $-0.583 * *$ & $-0.503 * *$ & -0.277 & $-0.436 *$ & $0.639 * *$ & $-0.579 * *$ \\
\hline $4 \mathrm{st}$ & $0.542 * *$ & $-0.468 * *$ & $-0.505 * *$ & -0.186 & -0.272 & $0.583 * *$ & $-0.483 * *$ \\
\hline
\end{tabular}

$* * p<0.01 * p<0.05$

Note) Spearman's Rank-Order Correlation Coefficient is calculated.

half: $\left.r=0 .-380^{*}\right)$. Error rates in the VDT task correlate with number of days in Task 1and Task 3.

\section{Correlation between VAS and task performance}

VAS scores (fatigue) fell off steadily along with the elapsed time (Fig. 2). Table 3 shows that the VAS scores (fatigue) of "1st" and "2nd" did not correlate with almost all task performance but "3rd" correlated with all task performance except for error rate in Task 2. Also, in the many other VAS scores, only "3rd" and "4th" scores correlated with plural task performances.
Correlation between physiological indices and task performance

There were correlations between SPL and calculation speed in the mental calculation of the first half $\left(\mathrm{r}=0.490^{* *}\right)$ and the second half $\left(\mathrm{r}=0.630^{* *}\right)$. Only BF correlated with error rate in the mental calculation of the second half $\left(\mathrm{r}=0.475^{* *}\right)$. Also, AAC correlated with error rate in the mental calculation of the second half $\left(\mathrm{r}=-0.525^{* *}\right)$ (Figs. 3, 4). As for the VDT task, there are correlations between SPL and error rate in Task 1 $\left(\mathrm{r}=0.404^{*}\right)$ and Task $3\left(\mathrm{r}=0.390^{*}\right)$. Also, there are correlations between AAC and error rate in Task $2\left(r=0.452^{*}\right)$ and Task 3 $\left(\mathrm{r}=0.422^{*}\right)$. 


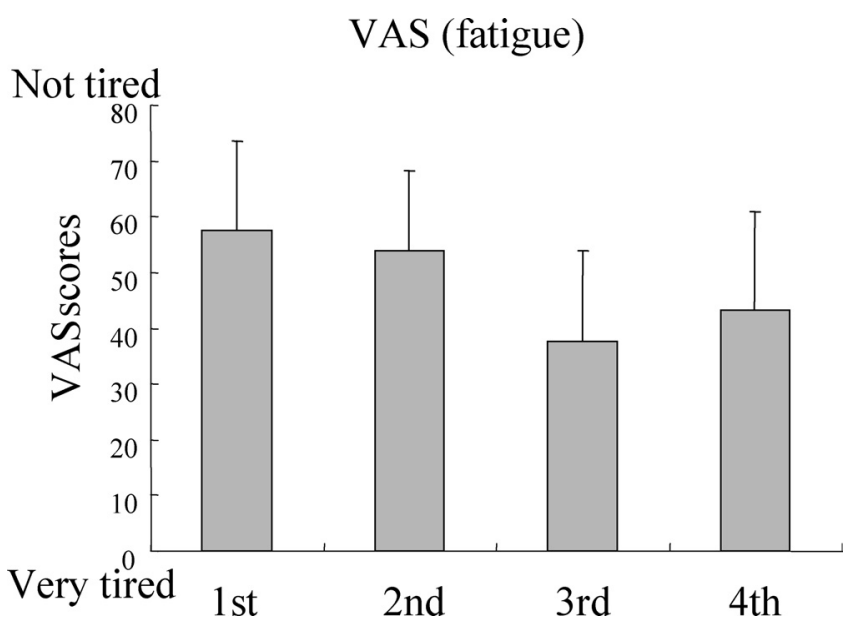

Fig. 2 VAS scores (fatigue) change with time.

Note) There were significant differences between all items (Wilcoxon signed ranks test).

\section{Discussion}

It is supposed that subjects became experienced as the days elapsed. Results suggest that task error rate is related with arousal level with accomplishment of the mental task. In contrast, task speed is not related with arousal level but proficiency of the mental task. This experiment shows the correlations between error rate and $\mathrm{BF}$, and also between calculation speed and SPL. These findings suggest that two kinds of task performance (calculation speed and error rate) can be recognized as having different characteristics in the mental task. Additionally, Takae et al. (2005) indicate that tissue blood volume at the tip of nose would be a useful index for measuring a driver's mental workload in driving. Also, it is reported that task difficulty can be evaluated by Electrodermal Activity (Kawasaki et al., 1994; Yoda et al., 2005). These papers support this experiment result, so it shall be assumed that SPL and BF are applied as a useful tool of evaluation in the mental task. VAS suggests that these correlations appeared according to the fatigue of the task.

In conclusion, this study suggested the hypothesis that the calculation speed and the error rate, which are considered as two different aspects of the task performance, are useful for evaluating task performance by evaluating them during one task and are supposed to be related to the different physiological background. In this study, SPL and BF were used to figure out different physiological background. Actually physiological indices show different response variability corresponding to every type of task (Callister et al., 1992; Kilseop and Rohae, 2005). In fact proficiency, arousal level, fatigue, task difficulty, and so on could be reflected in task performance. Calculation speed correlated with proficiency in this experiment, so a comparison between "be experienced" and "be inexperienced" is needed to estimate the relation between task performance and proficiency in plural types of task. This will be the subject of a further study. In order to

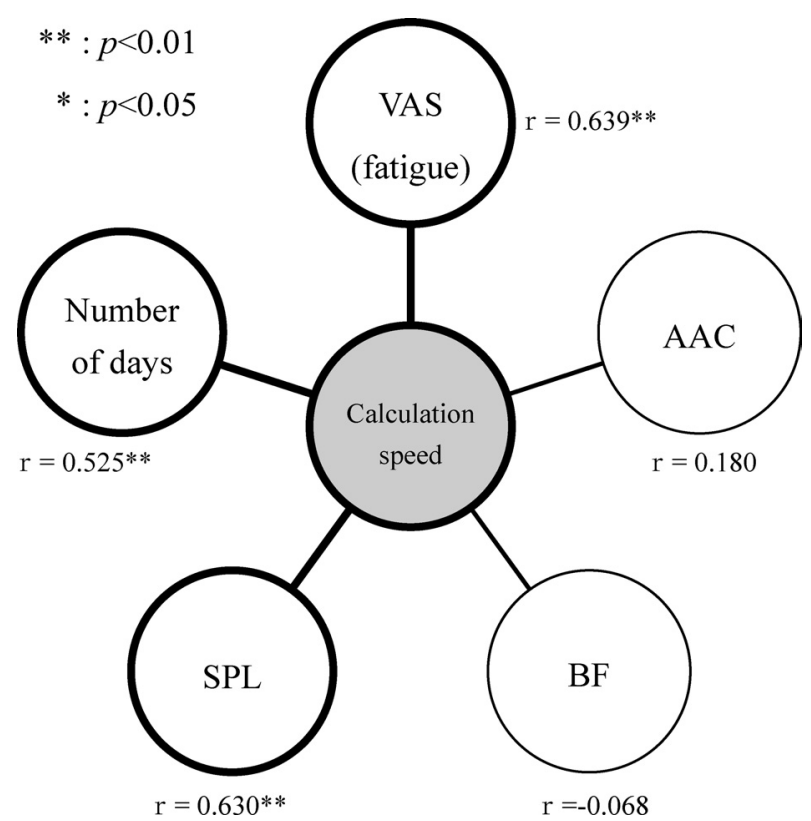

Fig. 3 Correlation between the calculation speed and other indices in the mental calculation of the second half.

Note) " $r$ " is Spearman's Rank-Order Correlation Coefficient.

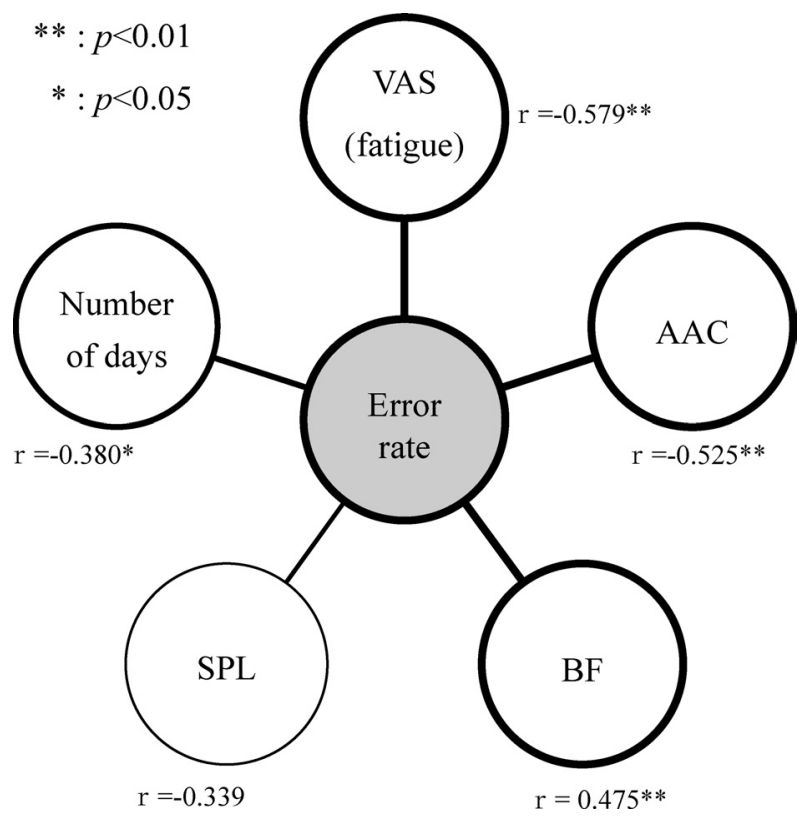

Fig. 4 Correlation between the error rate and other indices in the mental calculation of the second half.

Note) "r" is Spearman's Rank-Order Correlation Coefficient.

consider interindividual difference (Miyake, 1997), the number of measurements of one person has to be increased to observe the intraindividual variability in detail (Miyata et al., 1985). Applications relating to proficiency and intraindividual variability are currently under study. We will then attempt to do a more specific analysis for measurement data of one person. 
Acknowledgments We are grateful to all the subjects who cooperated and participated in this study. We are also grateful to our colleagues who participated in measurements. We are thankful to everybody in the laboratory that gave advice on the experiment.

\section{References}

Callister R, Suwarno NO, Seals DR (1992) Sympathetic activity is influenced by task difficulty and stress perception during mental challenge in humans. J Physiol 454: 373-387

Fumoto N (1977) Concentration on a task and change in pulse rate and finger skin blood flow. Jpn J Psychol 48: 289-295 [In Japanese]

Jin H, Shimomura Y, Iwanaga K, Katsuura T, Sugiura K, Mochzuki M (2005) Evaluation of the mental workload during a visual search task with working memory by various physiological indices. Jpn J Physiol Anthropol 10: 169-176 [In Japanese]

Kilseop R, Rohae M (2005) Evaluation of mental workload with a combined measure based on physiological indices during a dual task of tracking and mental arithmetic. Int $\mathrm{J}$ Ind Ergon 35: 991-1009

Kawasaki M, Konishi T, Takashima S, Sakaguchi M, Kamei T, Nakashima K, Ohhashi T (1994) The evaluation for skill of fractional calculations by emotional sweating rate. Tech Rep Inst Electr Inf Communic Engineers (ET): 94-111 [In Japanese]

Moore-Ede M (1993) The Twenty Four Hour Society. A William Patrick Book, California, 44-50

Michimori A, Stone P, Aguirre A, Stampi C (1994) Analysis of
Alpha Attenuation Test. J Sleep Res 23: 454 [In Japanese]

Michimori A, Araki K, Inbe H, Nakano T, Hagiwara H (1999)

Quantitative Assessment of the Alertness Levels during Sleep Deprivation using an EEG Spectral Analysis. Proc Symp Biol Physiol Eng 14: 397-400 [In Japanese]

Miyake S (1997) Factors Influencing Mental Workload Indexes. J UOEH 19: 313-325

Miyata Y, Fujisawa K, Kakigi T (1985) Physiological Psychology. Asakura-shoten, Tokyo, 253-259 [In Japanese]

Nishimura C, Nagumo J (1980) Biofeedback of Skin Potential Level for Doze Suppression. Iyodenshi To Seitai Kogaku 18: 327-333 [In Japanese]

Takae Y, Etori N, Watanabe T, Kubo N, Tozawa M, Miyake S (2005) A Study of Physiological Indices for Evaluating Drivers' Mental Workload. Annual meeting Jpn Soc of Mech Eng 2005: 315-316

Yagi A (1997) Modern psychology series 6. Cognition and perception. Baifu-kan, Tokyo, 72-83 [In Japanese]

Yoda T, Sugiura K, Horiguchi A, Shimomura Y, Iwanaga K, Katsuura T (2005) Evaluation of mental workload using electrodermal activity. Jpn J Physiol Anthropol 10: 134-135 [In Japanese]

Received: September 30, 2006

Accepted: July 24, 2007

Correspondence to: Emi Koyama, Graduate School of Science and Technology, Kyoto Institute of Technology, Matsugasaki, Sakyo-ku, Kyoto 606-8585, Japan

Phone: +81-75-724-7478

Fax: +81-75-724-7478

e-mail: koyama28@kit.ac.jp 